\title{
Surgical procedures and postsurgical tissue processing significantly affect expression of genes and EGFR-pathway proteins in colorectal cancer tissue
}

\author{
Kerstin A. David ${ }^{1}$, Florian T. Unger ${ }^{1}$, Philipp Uhlig ${ }^{1}$, Hartmut Juhl ${ }^{1}$, Helen M. \\ Moore $^{2}$, Carolyn Compton ${ }^{3}$, Björn Nashan ${ }^{4}$, Arnulf Dörner ${ }^{5}$, Andreas de Weerth ${ }^{5}$ \\ and Carsten Zornig ${ }^{6}$ \\ ${ }^{1}$ Indivumed GmbH, Hamburg, Germany \\ 2 Biorepositories and Biospecimen Research Branch, National Cancer Institute, National Institutes of Health, Bethesda, MD \\ ${ }^{3}$ Arizona State University, Phoenix, AZ \\ ${ }^{4}$ Clinic for Hepatobiliary Surgery and Transplantation Surgery, University Hospital Hamburg Eppendorf, Hamburg, Germany \\ ${ }^{5}$ Clinic for General and Visceral Surgery and Clinic for Gastroenterology, Agaplesion Diakonieklinikum Hamburg, Hamburg, \\ Germany \\ ${ }^{6}$ Surgical Clinic, Israelitisches Krankenhaus in Hamburg, Hamburg, Germany
}

Correspondence to: Hartmut Juhl, email: juhl@indivumed.com

Keywords: Biospecimen, predictive biomarker, drug development, tissue quality, EGFR pathway, phosphoproteins

Received: July 29, $2014 \quad$ Accepted: November 03, $2014 \quad$ Published: November 03, 2014

This is an open-access article distributed under the terms of the Creative Commons Attribution License, which permits unrestricted use, distribution, and reproduction in any medium, provided the original author and source are credited.

\section{ABSTRACT}

An understanding of tissue data variability in relation to processing techniques during and postsurgery would be desirable when testing surgical specimens for clinical diagnostics, drug development, or identification of predictive biomarkers.

Specimens of normal and colorectal cancer (CRC) tissues removed during colon and liver resection surgery were obtained at the beginning of surgery and postsurgically, tissue was fixed at 10, 20, and 45 minutes. Specimens were analyzed from 50 patients with primary CRC and 43 with intrahepatic metastasis of CRC using a whole genome gene expression array. Additionally, we focused on the epidermal growth factor receptor pathway and quantified proteins and their phosphorylation status in relation to tissue processing timepoints.

Gene and protein expression data obtained from colorectal and liver specimens were influenced by tissue handling during surgery and by postsurgical processing time. To obtain reliable expression data, tissue processing for research and diagnostic purposes needs to be highly standardized.

\section{INTRODUCTION}

The development of personalized medicine in oncology (determining an individual's disease risk, prognosis, and therapeutic options) is fostered by high-throughput analysis of molecular biomarkers in human cancer biospecimens [1]. Insufficient quality of such specimens may lead to spurious results and data misinterpretation [2]. Biospecimen quality depends on the pre-analytical conditions in which it was acquired [3]. Of critical importance is the time interval between reducing blood supply and removing the tissue (warm ischemia time), and the time interval between removing the tissue and preserving its molecular composition (cold ischemia time) [4-8]. In addition, patients (cells) are exposed to drugs and/or are manipulated in ways that may influence expression profiles and pathway activity, resulting in inaccurate analytical data. However, there are no systematic studies analyzing the impact of preanalytical factors.

The present study was conducted to gain a better understanding of the effects that warm and cold ischemia have on the molecular composition of a tissue specimen.

Specimens from normal and colorectal cancer (CRC) tissue, normal liver and intrahepatic metastases of CRC were collected in a highly standardized manner 
by specially trained staff present during entire surgery, both before surgery (presurgery), after hepatic pedicle clamping (post-clamping), and at 10, 20, and 45 minutes after resection of the tumor (10', 20', and 45' postsurgery, respectively). The molecular composition was investigated on the RNA, protein, and protein phosphorylation levels comparing normal tissue (with a rather homogeneous cell composition) with cancer tissue (representing a highly heterogeneous tissue with different ratios of malignant and [varying subtypes] of non-malignant cells).

The ultimate goals of this study were to:

i) Use whole genome gene expression analysis to provide a list of genes whose expression was unstable under warm and cold ischemic conditions and would need to be analyzed with caution in research and development programs.

ii) Determine variability of epidermal growth factor receptor (EGFR)-pathway proteins and their phosphorylation status with respect to tissue processing as examples of critical clinical biomarkers whose expression and activity level inform targeted therapy evaluation in cancer.

iii) Identify genes and proteins whose expression significantly changes during and after surgery, and therefore may serve as biomarkers of tissue quality.

\section{RESULTS}

\section{Patient recruitment}

Fifty patients with primary CRC and 43 with intrahepatic metastasis of CRC were enrolled in the study. From the 50 patients with a primary tumor, 370 formalinfixed paraffin-embedded (FFPE) and 780 frozen in liquid nitrogen (FF) tissue samples were collected, and from the 43 patients with metastasized cancer, 592 FFPE and 642 FF tissue samples were collected. All samples were subjected to morphological quality control (Supplementary Figure 1).

\section{Surgery and postsurgical tissue processing significantly affects gene expression in normal colon, normal liver, and CRC tissue}

Ten-minute clamping time of the hepatic artery significantly changed the expression of up to 690 (mean 118) genes in normal liver. The number of affected genes increased with surgery time (Figure 1). While some genes normalized (vs first biopsy), other genes had significant changes in expression level with prolonged surgery and postsurgical tissue processing time. The number of affected genes in normal liver and normal colon was similar (Table 1). In contrast to normal tissue, the variability of gene expression in relation to surgery and postsurgical processing time was significantly higher in cancer tissue. Within $10^{\prime}$ and $45^{\prime}$ 'postsurgery, up to 3,087 (mean 830) genes in metastatic liver CRC tumors showed $\geq 2$-fold and significant difference in expression. In primary $\mathrm{CRC}$ tissue, comparison between presurgery and 10' postsurgery biopsies identified up to 3,792 (mean 1,234) genes and $45^{\prime}$ postsurgery biopsies identified up to 4,116 (mean 1,553) genes. Supplementary Tables $1-4$ summarize all genes that showed a significant and $\geq 2$ fold change in expression during surgery and postsurgical processing.

\section{Identification of potential RNA-based tissue quality biomarkers}

Hierarchical clustering was used to further evaluate gene expression data and to categorize different patient groups. Clustering of data from patients who had colon surgery (normal and tumor tissue clustering separately) resulted in seven different partitions. Most patients $(89 \%$ with normal tissue) fell into the partition (presurgery/10', 20 ', 45' postsurgery) meaning that the presurgery timepoint separated from the $10^{\prime}, 20^{\prime}$, and $45^{\prime}$ postsurgery timepoints.

Excluding five patients who were regarded as outliers, only patients following the partition presurgery/10' $20^{\prime} 45^{\prime}$ postsurgery in normal tissue were used to compare gene expression intensity levels with multiple t-tests between the presurgery and 10' postsurgery timepoints, and presurgery and $45^{\prime}$ postsurgery timepoints. In normal colon tissue, 70 probes showed a differential expression in both comparisons. Of these, seven probes (encoding for five different genes) had a log-fold change of $\geq 2$ in both comparisons (Table 2). Expression of another five genes showed $\geq 2$ log-fold change in the comparison presurgery versus $45^{\prime}$ postsurgery only, while the log-fold change was slightly lower in the presurgery versus 10' postsurgery comparison. Genes that were significantly upregulated upon resection of normal colon tissue comprised transcription factors ( $E G R 1, F O S)$, signaling molecules (CYR61, RGS1, SGK1) of the extracellular matrix, and dual specificity phosphatase 1 ( $D U S P 1$, a protein that dephosphorylates MAPK1). Gene expression for mucosal proteins such as dual oxidase 2 (DUOX2, a protein that plays a role in antimicrobial defense), solute carrier family 6 (SLC6A14, a protein mediating amino acid transport), and vanin 1 ( VNN1, a protein involved in vitamin B5 recycling), was down-regulated. In colon tumor tissue, similar gene expression changes were found for CYR61, RGS1, DUSP1, DUOX2, and SLC6A14, although the log-fold change was generally lower compared to normal tissue (Table 2).

The same approach was not applicable to normal liver tissue because of the low number of affected genes and a more diverse change of expression (data not shown). 
Table 1: Differentially expressed genes in normal colon and liver tissues. Normal colon tissue is shown above the line and normal liver tissue shown below the line. Expression recorded: pre, before hepatic pedicle clamping; post, after clamping; 10', 10 minutes after resection; and 45', 45 minutes after resection. ${ }^{*} \mathrm{p}<0.05$.

\begin{tabular}{|c|c|c|c|c|}
\hline Probe ID & Gene & Protein & Pre vs 10' & Pre vs 45' \\
\hline 1555827 at & CCNL1 & Cyclin L1 & * & $*$ \\
\hline 201324 at & \multirow{2}{*}{ EMP1 } & \multirow{2}{*}{ Epithelial membrane protein 1} & * & $*$ \\
\hline 201325_s_at & & & * & \\
\hline 202499_s_at & $S L C 2 A 3$ & $\begin{array}{l}\text { Solute carrier family } 2 \text { (facilitated glucose transporter), } \\
\text { member } 3\end{array}$ & $*$ & $*$ \\
\hline 202672 s_at & ATF3 & Activating transcription factor 3 & * & \\
\hline 202988 s_at & \multirow{2}{*}{$R G S 1$} & \multirow{2}{*}{ Regulator of G-protein signaling 1} & $*$ & $*$ \\
\hline 216834 at & & & $*$ & $*$ \\
\hline 215034_s_at & TM4SF1 & Transmembrane 4 L six family member 1 & * & $*$ \\
\hline 227697 at & SOCS3 & Suppressor of cytokine signaling 3 & $*$ & $*$ \\
\hline 232304_at & PELII & Pellino homolog 1 (Drosophila) & $*$ & $*$ \\
\hline 36711_at & $M A F F$ & $\begin{array}{l}\text { V-maf musculoaponeurotic fibrosarcoma oncogene } \\
\text { homolog F (avian) }\end{array}$ & $*$ & \\
\hline 202291_s_at & $M G P$ & Matrix GLA protein & & $*$ \\
\hline 209101_at & $C T G F$ & Connective tissue growth factor & & $*$ \\
\hline 228335 at & CLDN11 & Claudin 11 & & $*$ \\
\hline 202859 x_at & IL8 & Interleukin 8 & & $*$ \\
\hline 228528 at & LOC 100286909 & Hypothetical protein LOC100286909 & & $*$ \\
\hline
\end{tabular}

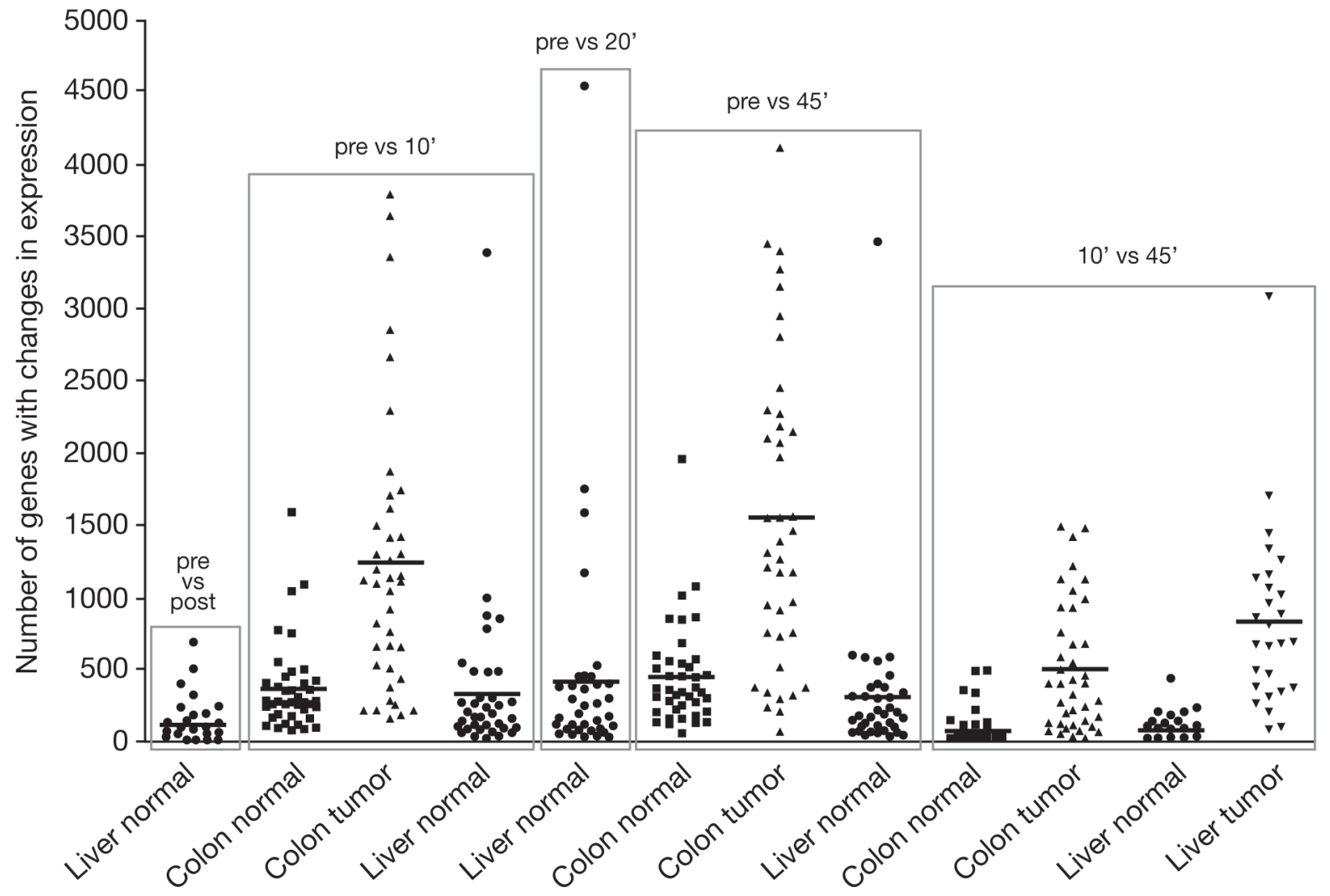

Figure 1: The variability of gene expression changes between patients, tissue type, surgery, and tissue processing times. The figure shows the number of genes whose expression changed by more than 2-fold according to the tissue source and timing of pedicle clamping and postsurgical processing: pre, before hepatic pedicle clamping; post, after clamping; 10', 10 minutes after resection; 20', 20 minutes after resection; and 45', 45 minutes after resection. Bars represent mean numbers of gene expression changes. 
Table 2: Differentially expressed genes in normal and colorectal tumor tissue. Gene expression was compared: pre, before hepatic pedicle clamping; post, after clamping; 10', 10 minutes after resection; 20', 20 minutes after resection; and 45', 45 minutes after resection.

\begin{tabular}{|c|c|c|c|c|c|c|c|c|c|c|}
\hline \multirow[b]{2}{*}{ Probe ID } & \multirow[b]{2}{*}{ Gene } & \multirow[b]{2}{*}{ Protein and function } & \multicolumn{2}{|c|}{$\begin{array}{l}\text { Normal colon tissue } \\
\text { Pre vs 10, }\end{array}$} & \multicolumn{2}{|l|}{ Pre vs 45, } & \multicolumn{2}{|c|}{$\begin{array}{l}\text { Colorectal tumor tissue } \\
\text { Pre vs 10' }\end{array}$} & \multicolumn{2}{|l|}{45 , } \\
\hline & & & p-value & $\begin{array}{l}\text { Log-fold } \\
\text { change }\end{array}$ & p-value & $\begin{array}{l}\text { Log-fold } \\
\text { change }\end{array}$ & p-value & $\begin{array}{l}\text { Log-fold } \\
\text { change }\end{array}$ & p-value & $\begin{array}{l}\text { Log-fold } \\
\text { change }\end{array}$ \\
\hline 201289_at & CYR61 & $\begin{array}{l}\text { Cysteine-rich angiogenic inducer } 61 \text {; extracellular matrix- } \\
\text { associated signaling protein that plays important roles in tissue } \\
\text { repair }\end{array}$ & $1.57 \mathrm{E}-18$ & 2.49 & $2.84 \mathrm{E}-22$ & 2.55 & 0.00000133 & 1.41 & $3.96 \mathrm{E}-08$ & 1.59 \\
\hline 202988_s_at & RGSI & Regulator of G-protein signaling 1; attenuates signaling activity & $4.21 \mathrm{E}-21$ & 2.34 & $5.05 \mathrm{E}-25$ & 2.39 & $1.81 \mathrm{E}-09$ & 1.57 & $7.62 \mathrm{E}-12$ & 1.91 \\
\hline 216834_at & & of G-proteins & $1.65 \mathrm{E}-22$ & 2.33 & $8.96 \mathrm{E}-25$ & 2.36 & $3.06 \mathrm{E}-09$ & 1.42 & $5.2 \mathrm{E}-12$ & 1.76 \\
\hline 201694_s_at & EGRI & Early growth response 1 ; transcription factor & $1.92 \mathrm{E}-12$ & 2.02 & $1.79 \mathrm{E}-18$ & 2.26 & . & . & . & . \\
\hline 227404 s_at & & & $1.4 \mathrm{E}-10$ & 1.98 & $2.57 \mathrm{E}-16$ & 2.32 & . & . & . & : \\
\hline 201739_at & SGKI & $\begin{array}{l}\text { Serum/glucocorticoid regulated kinase 1; activates potassium, } \\
\text { sodium and chloride channels }\end{array}$ & 4.03E-14 & 2.09 & $2.74 \mathrm{E}-16$ & 2.21 & . & . & . & . \\
\hline 209189_at & FOS & $\begin{array}{l}\text { FBJ murine osteosarcoma viral oncogene homolog; transcription } \\
\text { factor involved in cell proliferation, differentiation, survival, } \\
\text { hypoxia and angiogenesis }\end{array}$ & $1.26 \mathrm{E}-18$ & 2.77 & $9.3 \mathrm{E}-25$ & 3.19 & . & . & . & . \\
\hline 218541_s_at & C8orf4 & Chromosome 8 open reading frame 4 ; uncharacterized protein & $2.39 \mathrm{E}-12$ & 2 & $3.64 \mathrm{E}-15$ & 2.01 & . & . & & \\
\hline 201041_s_at & DUSPI & $\begin{array}{l}\text { Dual specificity phosphatase 1; dephosphorylates MAP kinase } \\
\text { MAPK1/ERK2 }\end{array}$ & $1.21 \mathrm{E}-17$ & 1.85 & $3 \mathrm{E}-23$ & 2.15 & . & . & 0.0000115 & 1.14 \\
\hline 219727_at & DUOX2 & $\begin{array}{l}\text { Dual oxidase 2; plays a role in antimicrobial defense at the } \\
\text { mucosal surface }\end{array}$ & $3.36 \mathrm{E}-08$ & -1.88 & 2.24E-10 & -2.14 & 0.000497 & -1.51 & 0.0000133 & -1.82 \\
\hline 219795_at & SLC6A14 & $\begin{array}{l}\text { Solute carrier family } 6 \text { (amino acid transporter), member 14; } \\
\text { mediates the uptake of a broad range of amino acids }\end{array}$ & 0.0000101 & -1.87 & 0.00000036 & -2.16 & 0.0109 & -1.2 & 0.000316 & -1.75 \\
\hline 205844_at & $V N N I$ & $\begin{array}{l}\text { Vanin 1; amidohydrolase recycling pantothenic acid (vitamin } \\
\text { B5) and releasing cysteamine }\end{array}$ & 0.000102 & -1.62 & 0.000000164 & -2.17 & . & . & . & . \\
\hline
\end{tabular}

\section{Identification of housekeeping genes not affected by surgery and tissue processing}

Gene expression data were sorted according to the coefficients of variance $(\mathrm{CV})$. The 10 probe sets with the lowest $\mathrm{CV}$ across all four timepoints in normal tissue are listed in Table 3. While some probe sets encoded for insufficiently explored proteins, among those very constitutively-expressed genes was eukaryotic translation elongation factor 1 alpha 1 (EEF1A1), a widely expressed gene with high copy numbers that is known as a potential housekeeping gene for gene expression analysis [9]. Further well-known housekeeping genes with low CV were ribosomal proteins L13 and S18, beta-glucuronidase, and beta-actin, while other frequently used housekeeping genes, such as beta-2-microglobulin and beta2B-tubulin, were not constitutively expressed.

The same exercise was conducted for gene expression in CRC tissue (data not shown). Again, EEF1A1 expression showed a very low $\mathrm{CV}$, suggesting it may function as a reference gene in both normal and neoplastic colorectal tissue.

\section{Up to $60 \%$ of patients showed changes of EGFR- pathway protein expression}

EGFR and its downstream key signaling proteins of the AKT and MAPK pathway were investigated in relation to total protein concentration and phosphorylation status. Using expression levels of presurgery biopsies as a reference, changes (up or down) in protein expression of $\geq 2$-fold were documented. In normal liver tissue the preclamping tissue biopsy was used as reference.

Overall, changes in the expression of EGFRpathway proteins were lowest in normal liver, higher in normal colon, and highest in cancer tissue (Supplementary Figure 2). In liver tissue, a change in total protein expression was not observed, while in normal colon tissue EGFR expression changed $\geq 2$-fold in $10 \%$ of patients, while expression of the downstream protein p 70 -S6K changed $>2$-fold in $35 \%$ of patients between presurgery and $45^{\prime}$ postsurgery. In CRC tissue, expression changes in EGFR-related proteins were striking. Between presurgery and 10' postsurgery, EGFR total protein expression changed by $>2$-fold in $20 \%$ of all patients and in $30 \%$ of all patients within a further 35 minutes (presurgery $/ 45$ ' postsurgery). Subsequently, expression of downstream proteins, such as p70-S6K, changed $>2$-fold from presurgery to 45 ' postsurgery in more than $60 \%$ of patients.

While the change in total protein level became statistically significant for AKT, MTOR, ERK $1 / 2$, GSK3B, p70-S6K (Figure 2), and HIF1A (data not shown), other proteins (Figure 2) such as EGFR showed up- and down-regulation in individual patients and, while still showing unstable expression in some patients, it was not statistically significant (Supplementary Figure 3).

\section{Surgery and postsurgical processing strongly affects phosphorylation of key signaling molecules within the AKT and MAPK pathway}

The phosphorylation status of key signaling proteins was significantly affected in most patients and to a larger extent in tumor tissue compared with normal tissue. We found a chain of phosphorylation events indicating activation in some and inactivation in other parts of the phosphorylation cascade (data not shown). While these changes remain to be evaluated by a more detailed analysis of clinical variables, we found statistically significant changes in most key regulatory proteins between presurgery and 10' postsurgery samples and additional changes for some proteins during the postsurgical cold ischemia time. This included AKT, MTOR, ERK1/2, and MEK (Figure 2).

In normal colon and liver tissue, total levels of most proteins did not differ significantly between timepoints. 
Table 3: The 10 probe sets ( 9 genes) with the lowest coefficient of variation (CV) across all four timepoints (presurgery and 10, 20, and 45 minutes after resection) in normal colon tissue are shown above the dotted line, and 20 well-known housekeeping genes (HKG) with their CV and their rank when sorted for CV are shown below the dotted line.

\begin{tabular}{|c|c|c|c|c|c|}
\hline \multirow{2}{*}{ Probe ID } & \multirow{2}{*}{ Gene } & \multirow{2}{*}{ Protein and function } & \multicolumn{2}{|l|}{ Normal tissue } & \multirow{2}{*}{$\begin{array}{l}\text { Tumor tissue } \\
\text { Rank }\end{array}$} \\
\hline & & & $\mathbf{C V}$ & Rank & \\
\hline 1558623 at & LOC729121 & Hypothetical LOC729121 & 0.0000651769 & 1 & 22,547 \\
\hline 227472_at & $D D A 1$ & $\begin{array}{l}\text { DET1 and DDB1 associated 1; may be } \\
\text { involved in ubiquitination and subsequent } \\
\text { proteosomal degradation }\end{array}$ & 0.0000763301 & 2 & 5,095 \\
\hline 213477_x_at & \multirow{2}{*}{ EEF1A1 } & \multirow{2}{*}{$\begin{array}{l}\text { Eukaryotic translation elongation factor } 1 \\
\text { alpha 1; involved in protein biosynthesis }\end{array}$} & 0.000120035 & 3 & 70 \\
\hline 206559 x_at & & & 0.00021194 & 10 & 464 \\
\hline 203172_at & $F X R 2$ & \begin{tabular}{|l|l|} 
Fragile X mental retardation, autosomal \\
homolog 2; RNA-binding protein
\end{tabular} & 0.00012495 & 4 & 5,833 \\
\hline 202652_at & $A P B B 1$ & $\begin{array}{l}\text { Amyloid beta (A4) precursor protein-binding, } \\
\text { family B, member 1; transcription coregulator }\end{array}$ & 0.000140598 & 5 & 22,323 \\
\hline 209394_at & $A S M T L$ & $\begin{array}{l}\text { Acetylserotonin } \text { O-methyltransferase-like; } \\
\text { unknown function }\end{array}$ & 0.000153558 & 6 & 28,176 \\
\hline 234891 at & DKFZP547L112 & Hypothetical protein DKFZp547L112 & 0.000198356 & 7 & 17,884 \\
\hline 212986_s_at & $T L K 2$ & $\begin{array}{l}\text { Tousled-like kinase } 2 \text {; involved in chromatin } \\
\text { assembly }\end{array}$ & 0.000208644 & 8 & 2,237 \\
\hline 223148_at & $P I G S$ & $\begin{array}{l}\text { Phosphatidylinositol glycan anchor } \\
\text { biosynthesis, class S; component of the GPI } \\
\text { transamidase complex }\end{array}$ & 0.000210952 & 9 & 24,752 \\
\hline $210646 \_\mathrm{x}$ at & RPL13A & Ribosomal protein L13a, HKG & 0.000370481 & 53 & 752 \\
\hline 201049 s_at & RPS18 & Ribosomal protein S18, HKG & 0.000581215 & 185 & 2,319 \\
\hline 230125 at & GUSB & Glucuronidase, beta, $\mathrm{HKG}$ & 0.000939722 & 647 & 19,340 \\
\hline $\begin{array}{l}\text { AFFX- } \\
\text { HSAC07/ } \\
\text { X00351_3 } \\
\text { at }\end{array}$ & $A C T B$ & Actin, beta, HKG & 0.001080551 & 941 & 7,550 \\
\hline 216457_s_at & SF3A1 & Splicing factor 3a, subunit 1, $120 \mathrm{kDa}, \mathrm{HKG}$ & 0.001542067 & 2,363 & 890 \\
\hline 212581_x_at & $G A P D H$ & $\begin{array}{l}\text { Glyceraldehyde-3-phosphate dehydrogenase, } \\
\text { HKG }\end{array}$ & 0.001735025 & 3,208 & 332 \\
\hline 201093_x_at & $S D H A$ & $\begin{array}{l}\text { Succinate dehydrogenase complex, subunit A, } \\
\text { flavoprotein (Fp), HKG }\end{array}$ & 0.002533378 & 8,062 & 42,630 \\
\hline 203135 at & $T B P$ & TATA box binding protein, $\mathrm{HKG}$ & 0.003036083 & 12,044 & 11,750 \\
\hline 203040 s_at & $H M B S$ & Hydroxymethylbilane synthase, HKG & 0.003145361 & 12,988 & 6,489 \\
\hline 1565446_at & HPRT1 & $\begin{array}{l}\text { Hypoxanthine phosphoribosyltransferase 1, } \\
\text { HKG }\end{array}$ & 0.003323065 & 14,616 & 23,933 \\
\hline 229165 at & MRPL12 & Mitochondrial ribosomal protein L12, HKG & 0.003428532 & 15,543 & 12,742 \\
\hline 224695 at & $C 2 \operatorname{orf} 29$ & Chromosome 2 open reading frame $29, \mathrm{HKG}$ & 0.00344027 & 15,651 & 6,081 \\
\hline 211296 x_at & $U B C$ & Ubiquitin $\mathrm{C}, \mathrm{HKG}$ & 0.003508935 & 16,259 & 2,040 \\
\hline 235741_at & PPIA & $\begin{array}{l}\text { Peptidylprolyl isomerase A (cyclophilin A), } \\
\text { HKG }\end{array}$ & 0.003666547 & 17,628 & 33,077 \\
\hline 221842 s_at & ZNF131 & Zinc finger protein $131, \mathrm{HKG}$ & 0.003960494 & 20,273 & 20,072 \\
\hline 227708_at & EEF1A1 & $\begin{array}{l}\text { Eukaryotic translation elongation factor } 1 \\
\text { alpha } 1, \mathrm{HKG}\end{array}$ & 0.004407467 & 24,160 & 24,765 \\
\hline 1566191_at & SUZ12 & $\begin{array}{l}\text { Suppressor of zeste } 12 \text { homolog (Drosophila), } \\
\text { HKG }\end{array}$ & 0.005051028 & 29,227 & 49,826 \\
\hline $240686 \_$__at & TFRC & Transferrin receptor $(\mathrm{p} 90, \mathrm{CD} 71), \mathrm{HKG}$ & 0.006000472 & 35,483 & 30,303 \\
\hline 232311_at & $B 2 M$ & Beta-2-microglobulin, HKG & 0.012872315 & 49,756 & 44,528 \\
\hline 214023 x_at & $T U B B 2 B$ & Tubulin, beta $2 \mathrm{~B}, \mathrm{HKG}$ & 0.019826709 & 52,506 & 53,778 \\
\hline
\end{tabular}



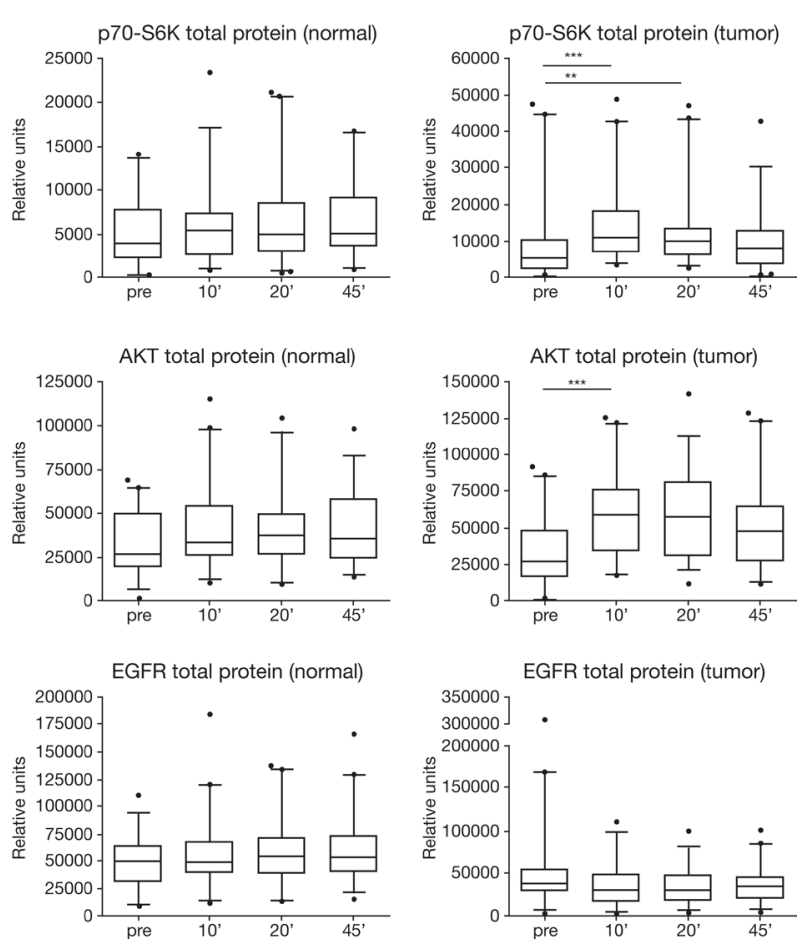

$2 b$
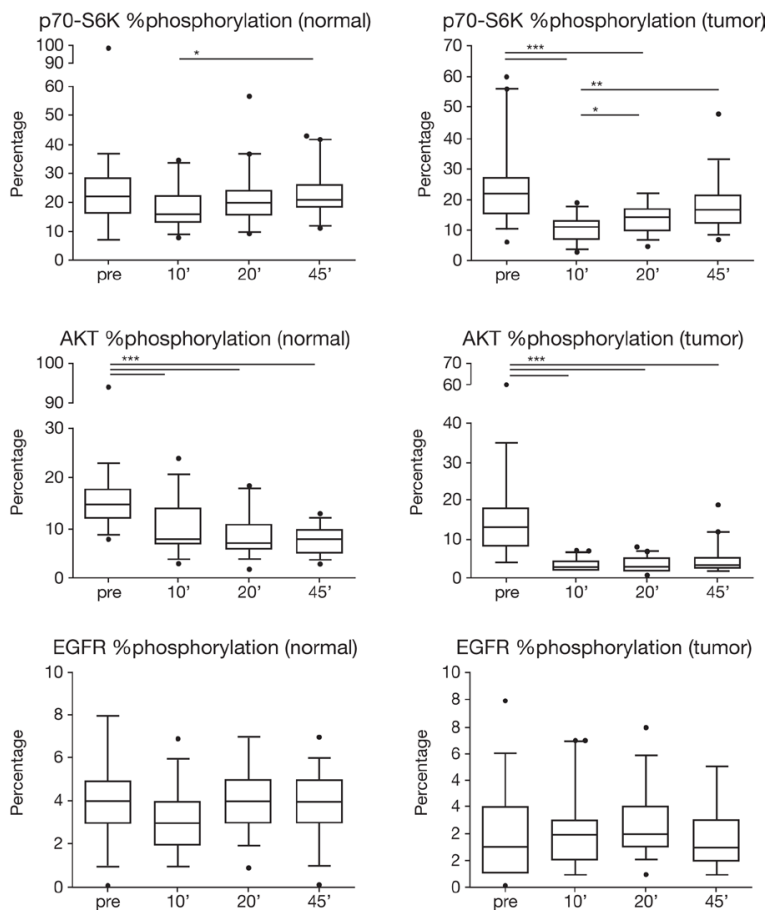
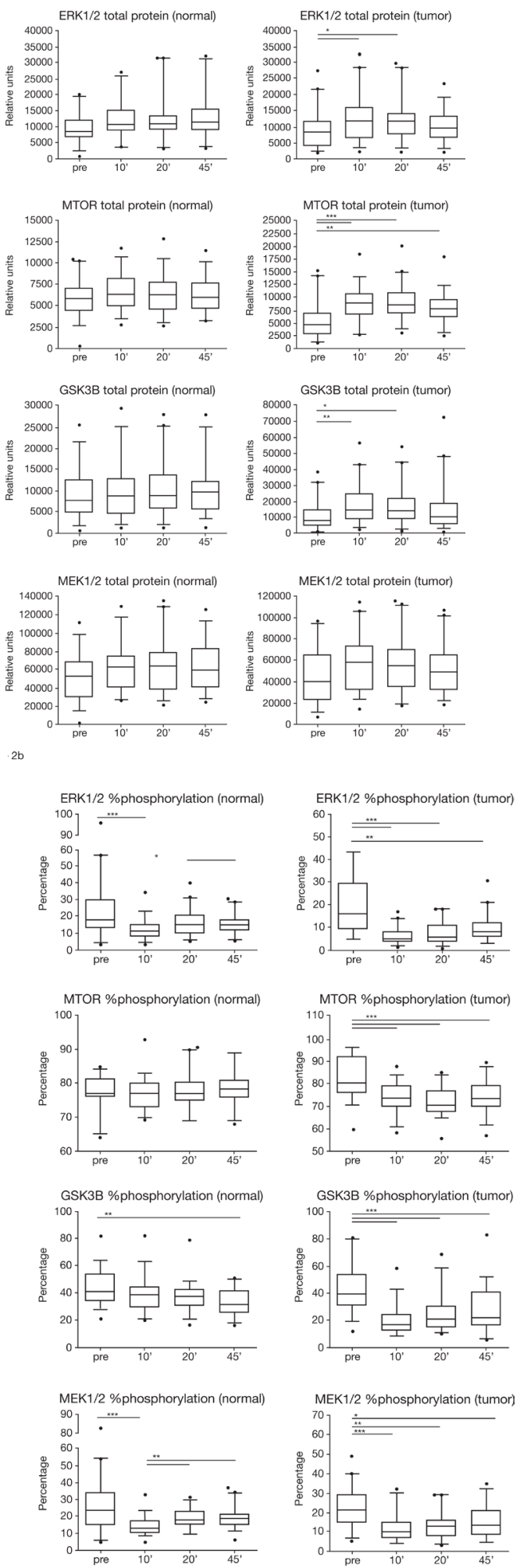

Figure 2 (A) Total protein expression (relative units) of p70-S6K, AKT, EGFR, ERK1/2, MTOR, GSK3B, and MEK1/2 in normal and tumor colon tissue at four timepoints of tissue collection: pre, before hepatic pedicle clamping; 10', 10 minutes after resection; 20', 20 minutes after resection; and 45', 45 minutes after resection. ${ }^{*} \mathrm{p}<0.05 ; * * \mathrm{p}<0.01 ; * * * \mathrm{p}<0.001$. Box plots indicate the 5/95\% confidence interval, median, and standard deviation. (B) Percentage of protein phosphorylation of p70-S6K, AKT, EGFR, ERK1/2, MTOR, GSK3B, and MEK1/2 in normal and tumor colon tissue at four timepoints of tissue collection: pre, before hepatic pedicle clamping; 10', 10 minutes after resection; 20', 20 minutes after resection; and 45', 45 minutes after resection. * $\mathrm{p}<0.05 ;{ }^{* *} \mathrm{p}<0.01$; $* * * p<0.001$. Box plots indicate the $5 / 95 \%$ confidence interval, median, and standard deviation. 
However, there was a statistically significant increase at 10' postsurgery for AKT and MTOR in CRC tissue. Protein phosphorylation decreased significantly with warm and cold ischemia in normal colon and CRC tumor tissue (Figure 2). It is important to note that protein phosphorylation showed similar trends for changes in selected proteins in both tissue types (normal tissue and highly heterogeneous tumor tissue). This implies that these regulations occur irrespective of the cell type and, thus, could serve as tissue quality marker independent of the kind of tissue (normal and tumor).

The above mentioned decline in protein phosphorylation was mostly associated with decreased stain intensity upon immunohistochemistry, which was statistically significant for phosphorylated EGFR, AKT, and ERK1/2. Example images of immunohistochemistry for the detection of $\mathrm{p}$-AKT in a patient with colon cancer in relation to ischemia time are shown in Figure 3.

\section{HSP27 phosphorylation increases with warm and cold ischemia}

HSP27 was evaluated as its expression is known to respond to cellular stress [10]. While total HSP27 protein

(A)

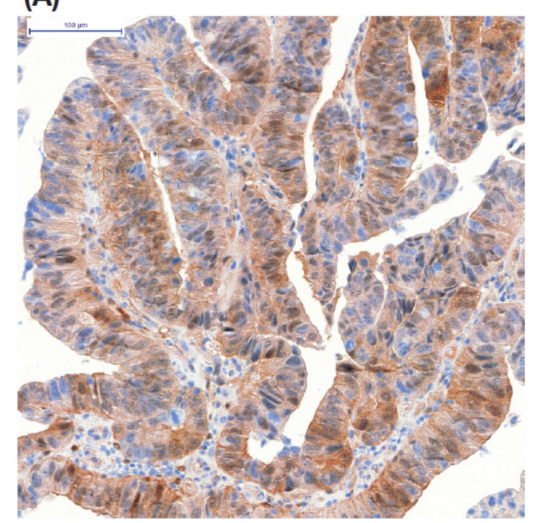

(C)

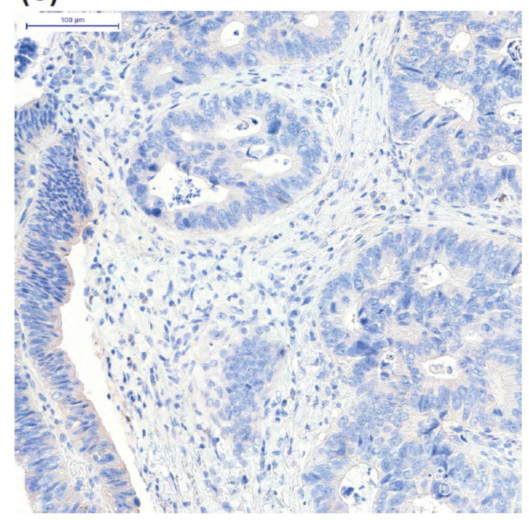

levels were similar across all groups, the percentage of phosphorylated versus total HSP27 increased over time in an almost linear fashion from presurgery to $45^{\prime}$ postsurgery in most patients, demonstrating a statistically significant difference between presurgery/preclamping samples versus those taken after prolonged cold ischemia (Supplementary Figure 4). After 45' postsurgery of cold ischemia, the proportion of HSP27 phosphorylation had increased 8 -fold compared to presurgery levels. In normal liver tissue it had increased 2-fold.

\section{DISCUSSION}

Pharmaceutical companies put significant efforts into the development of specific pathway inhibitors, and new drugs can emerge in high numbers from preclinical development programs. Identification of drug targets as stratification and predictive biomarkers in patient populations has become an important field in drug development; indeed, such biomarkers are thought to be essential for current and future patient care and an important strategy in controlling reimbursement costs in cancer care.

While DNA aberrations such as activating mutations in specific genes allow the identification to some extent of

(B)

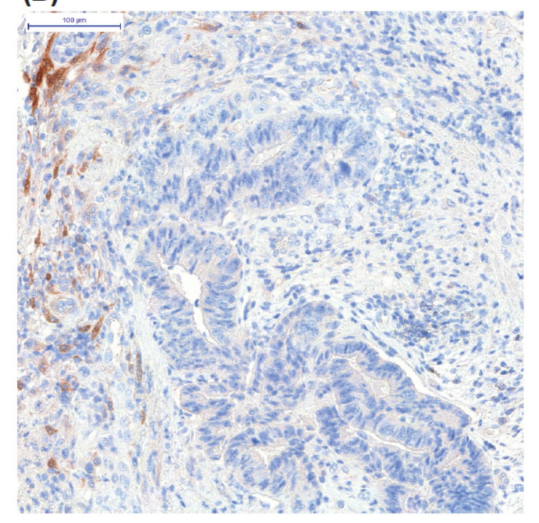

(D)

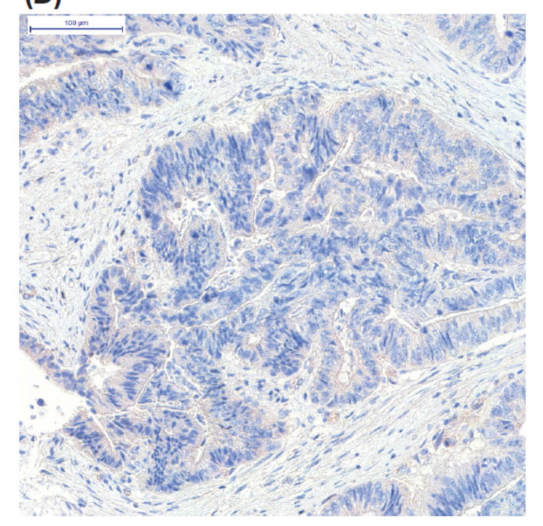

Figure 3: Representative immunohistochemistry for pAKT on formalin-fixed colon cancer tissue from one patient taken at four timepoints: (A) biopsy presurgery; (B) tissue fixed 10 minutes after resection; (C) tissue fixed 20 minutes after resection; and (D) tissue fixed 45 minutes after resection. 
drug targets under various circumstances, this approach is of limited predictive value. Utilization of protein targets and cancer pathway activity as determined by quantifying phosphorylation of regulatory proteins can provide a much deeper insight into a patient's individual tumor biology. However, a major challenge for the discovery and development of predictive drug targets is the need for tissue samples that truly represent the reality of a patient's tumor biology. As most tissues are surgical specimens, a major concern is the risk of modified expression levels because of tissue manipulation during and after surgery. Understanding the effects of surgical manipulation on cancer biomarkers will be an important part of the knowledge base upon which such biomarkers can be fully utilized to benefit patients.

Stemming the blood supply rapidly induces hypoxia and cellular stress and is thought to affect the molecular composition of cells and thus, the analytical data derived from these tissues. In addition, during surgery, patients (and, naturally, their cells) are exposed to various kinds of drugs given by the anesthetist.

In this study we demonstrate that human tissue is highly susceptible to surgical factors and prolonged postsurgical ischemia, and standardized handling of tissue is an important factor for subsequent analysis. Analyzing known stress and ischemia markers such as HSP27 [12] and HIF1A [13-14], we found that in normal liver tissue, HSP27 phosphorylation was significantly increased at 10 minutes after hepatic pedicle clamping and further increased throughout post-resection ischemia in a time-dependent manner. In normal colon tissue, HSP27 phosphorylation increased in an almost linear time-dependent manner between 10' and 45' postsurgery. Hence, HSP27 phosphorylation appears to be an interesting marker for pre- and post-resection tissue quality. We also found a significant increase in HIF1A protein levels $10^{\prime}$ postsurgery of colorectal tumors which, however, declined again at later timepoints. Interestingly, influence of ischemia on protein phosphorylation has been detected independent from tissue type (normal vs cancer) and the cellular composition of tumor tissue with respect to tumor cell content and non-malignant cell subtypes such as stroma cells and inflammatory infiltrates.

In individual patients the expression of more than 4,000 genes were altered and up to $60 \%$ of patients with primary CRC showed more than a 2-fold expression change in proteins and their phosphorylation, which is in agreement with previous studies demonstrating cold ischemia-related alterations in gene and protein expression $[4,8]$. In general, the impact on the molecular composition was more severe in tumor tissue compared to normal tissue, likely due to the higher activity of tumor cells compared to normal cells. The most striking changes were observed during warm ischemia and early cold ischemia (10' postsurgery); gene and protein expression changes during prolonged cold ischemia were surprisingly less prominent though still significant. Interestingly, up to 690 (mean 118) genes were already affected in individual patients by just clamping the hepatic pedicle for 10 minutes.

With regard to gene and protein expression data, we observed inter-individual variability across patients. Most of these patients, however, appeared to follow a similar pattern of gene expression changes. By only using those patients for further gene expression analyses, we were able to identify significant up-regulation of several transcription factors and signaling molecules of the extracellular matrix such as cysteine-rich angiogenic inducer 61 (CYR61, CCN1), and the regulator of G-protein signaling 1 (RGS1). CYR61 is a matrix cell-adhesion molecule. Depending on the context, it promotes cell proliferation, survival, apoptosis, or angiogenesis by binding to distinct integrins and plays an important role in wound repair [15]. RGS1 attenuates the signaling activity of G-proteins by fostering GTP hydrolysis and has various immunomodulatory functions [16]. Simultaneously, the expression of several genes from colonic enterocytes was down-regulated.

On the protein level, the variability between patients was also high. Protein levels and their phosphorylation status showed increasing and decreasing levels between different patients and timepoints. Variability was higher in tumor tissue, probably because of the highly heterogeneous cell composition, but also occurred in homogeneous normal tissue. This implies that the observed changes are to a large extent related to tissue processing and not just variability in tumor cell content. However, identification of tumor cell-specific events and cancer cell biomarker requires analysis of isolated cancer cells, which will be performed in follow-up studies.

However, the most consistent molecular change during tissue resection and post-surgical ischemia was a decrease in protein phosphorylation, observed for AKT, MTOR, p70-S6K, GSK3B, and ERK1/2 from colon tissue. Dephosphorylation of ERK1/2 is particularly interesting with regard to the fact that an up-regulated gene expression was found for dual specificity phosphatase 1 (DUSP1, MKP1). This protein can dephosphorylate MAPK (ERK) in the cell nucleus and thus attenuates MAPK signaling [17].

Even in our highly standardized tissue processing protocol, a comparison between frozen and formalin fixed tissue is difficult because the slow formalin penetration significantly prolongs the ischemia time (see Methods). However, the decline in phosphorylation status observed in frozen tissue had an influence on immunohistochemical staining for $\mathrm{pERK}, \mathrm{pAKT}$, and pEGFR in colon tissue, where staining intensity was often lower in postsurgery compared to presurgery samples. This has important consequences, since immunohistochemistry is frequently used to determine activation of signaling pathways in individual cancer specimens in order to determine personalized treatment options. The pathologist analyzing 
post-resection specimens needs to be aware of the alterations that can be induced by cold ischemia time and therefore needs precise information about the conditions under which the tissue was procured. Since DUSP1, CYR61, and RGS1 gene expression was up-regulated in both normal and CRC tissue upon tissue resection, these genes may represent interesting candidates for biomarkers of post-resection tissue quality. Further evaluations by RT-PCR and protein quantification; however, would be needed for their characterization and validation as quality biomarkers.

Our study also identified candidate "housekeeping" or relatively stable genes, the expression of which was not altered by tissue resection and post-surgical cold ischemia. In this regard, the EEF1A1 gene appears to be particularly interesting. Its $\mathrm{CV}$ was very low across all four timepoints, both in normal colon tissue and in CRC tissue. EEF1A1 is known to be constitutively expressed in many tissues and under various conditions and has been described as a useful housekeeping gene for gene expression analyses [18]. Further candidates for reference genes have been identified. Interestingly, genes that are frequently used as reference genes such as beta2-microglobulin or beta2tubulin did not show constitutive expression throughout resection and post-surgical ischemia and therefore do not appear suitable as housekeeping genes under the described conditions.

The data presented herein are largely in agreement with a recent report that described fluctuations of protein levels and protein phosphorylation in human intestine tissue samples as a consequence of different ischemic conditions before preservation [8]. The authors reported that a general trend towards up- or down-regulation of proteins was not evident due to pronounced interindividual variability. We were able to demonstrate a general trend towards up- and down-regulation of proteins and their phosphorylation status due to a higher number of samples that were investigated in our study, but confirm the occurrence of high inter-individual variability. In fact, we identified a majority of patients that responded towards tissue resection with a common pattern of gene expression changes, while some other patients responded in a completely different manner. These inter-individual differences require further investigation.

In summary, our data show a significant difference in the molecular composition of tissue specimens collected after tumor resection compared to specimens collected via colonoscopy before tumor resection. This difference is larger than the difference between various post-resection times between 10 and 45 minutes. The observed effect is either due to warm and cold ischemia, and/or the anesthesia/surgical procedure itself, and the manipulation of the tissue. The molecular changes induced during and after tumor resection are heteromorphic and do not occur in all patients. In general, kinase proteins become dephosphorylated, which may result in decreased intensity of immunohistochemical staining. The ratio between phosphorylated and total HSP27 protein has emerged as a promising marker for tissue quality, since it demonstrates an almost linear increase with prolonged cold ischemia time in all three tissue types.

This study presents an important contribution to the understanding of molecular changes that are being introduced into tissue samples during the pre-analytical phase, i.e. by the tissue collection procedure itself and the surgical procedures prior tissue collection. For the first time, we provide a full list of genes whose expression is altered due to tissue processing and surgical manipulation. In addition, our findings suggest that the analysis of regulatory pathway proteins and specific growth factor receptors, such as EGFR, requires the use of highly standardized and rapid tissue processing techniques. Once standardized techniques have been validated, analysis of the expression of regulatory pathway proteins may be valuable as predictive markers of targeted therapies.

\section{ONLINE METHODS}

\section{Patient enrollment}

Fifty patients with colorectal cancer (CRC) and 43 with hepatic metastasis of CRC who were scheduled for tumor resection surgery gave informed consent to be enrolled in the study. Only patients with a tumor larger than $3 \mathrm{~cm}$ in diameter were enrolled. Patients who had received chemotherapy or radiation therapy $<3$ weeks before surgery were excluded. Specially trained study nurses were present during all surgeries. They performed tissue processing in the surgical unit and clinical data documentation to assure that the same standardized operating procedures have been applied to all patients. The study was conducted at three sites in Hamburg, Germany, and received approval by the competent ethics review committee of the medical association Hamburg under reference no. PV3342.

\section{Tissue collection before and during surgery}

After induction of anesthesia, patients with primary CRC underwent a colonoscopy, upon which three biopsies were taken from the tumor and three biopsies from the adjacent normal tissue (presurgery) (Supplementary Figure 1). For patients with hepatic metastasis of CRC, four pieces of tissue were taken from normal liver parenchyma before the start of liver resection, i.e. just before clamping of the hepatic artery (presurgery). About 10 minutes after hepatic pedicle clamping (post-clamping), another four tissue samples were collected from the normal liver parenchyma (postsurgery).

For all patients, 12 tissue samples were each 
collected from the tumor tissue and the adjacent normal tissue after resection of the tumor and adjacent normal tissue. These 24 samples were divided into three groups, each exposed to a cold ischemia time of 10 minutes (10'), 20 minutes (20'), and 45 minutes (45'), respectively. To minimize variation because of formalin penetration time (approximately $1 \mathrm{~mm} /$ hour), every tissue sample had a similar size of approximately 5 x 5 x $5 \mathrm{~mm}$ and an approximate weight of $120 \mathrm{mg}$. Since formalin penetration is slow, samples were all fixed for 16 hours. For each timepoint and tissue type (normal or tumor), half of the tissues were immediately stored in the vapor phase of liquid nitrogen, while the other half was immersion fixed in 4\% buffered formaldehyde (Supplementary Figure 1).

\section{Processing of tissue specimens}

All tissue specimens in formaldehyde were immersion fixed for 16 to 72 hours. Thereafter, they were weighed and placed in $70 \%$ ethanol for a maximum of 24 hours until further processing. Processing was conducted with an automated system (Microm tissue processor STP 420 D Thermo Scientific, Dreieich, Germany) resulting in the embedding of tissues in paraffin (Paraplast).

From each formalin-fixed paraffin-embedded (FFPE) and frozen in liquid nitrogen (FF) tissue specimen, one section was stained with hematoxylin-eosin and evaluated under a light microscope in order to verify the presence of tumor and normal tissue, respectively. Tumor content was $10-90 \%$ in tumor samples and $0 \%$ in all adjacent normal samples.

After histological quality control, FFPE and FF samples were selected for the following molecular analyses: i) quantification of total and phosphorylated protein by a medium-throughput enzyme-linked immunosorbent assay technology (Meso Scale Discovery [MSD]); ii) semi-quantitative evaluation of protein expression by immunohistochemistry; and iii) gene expression profiling on total RNA extracts using an Affymetrix whole genome chip (Supplementary Figure 1).

\section{Quantification of proteins}

Forty FF specimens were used. Tissue lysates were prepared by cutting and homogenizing a $20 \mu \mathrm{m}$ slice from each FF specimen. The resulting tissue lysate was subjected to a bicinchoninic acid protein assay (BCA kit; Sigma, Steinheim, Germany) to determine protein concentration. Quantification of proteins was conducted using 96-well plates with capture antibodies based on the assay platform from MSD (Gaithersburg, MD, USA). The following assay kits were used: HIFlalpha singleplex, HSP27/pHSP27(Ser15) duplex, EGFR/pEGFR(Tyr1173) duplex, AKT/pAKT(Ser473) duplex, MTOR/ pMTOR(Ser2448) duplex, p70-S6K/pp70-S6K(Thr421,
Ser424) duplex, GSK3B/pGSK3B(Ser9) duplex, MEK1/2/pMEK1/2(Ser217/221) duplex and ERK1/2/ pERK1/2(Thr202/Tyr204, Thr185/Tyr187) duplex.

Assays were performed using $10 \mu \mathrm{g}$ of tissue lysate according to the manufacturers' instructions and analyzed with the SECTOR Imager platform (MSD). Analyses were conducted in triplicate and arithmetic mean values were calculated. Mean values of post-surgery samples were against presurgery samples from the same patient. The percentage of phosphorylation was calculated according to the formula: phosphorylation $(\%)=(\mathrm{DFx}$ phosphorylated protein)/(phosphorylated protein + total protein) $\times 100$ with distribution factor $(\mathrm{DF})=2$. If the phosphorylation (\%) was $>100$, DF was adjusted. In order to account for inter-assay variation, lysates of stimulated human cells were produced and employed as positive and negative controls.

\section{Immunohistochemistry}

$5 \mu \mathrm{m}$ sections from FFPE tissue were mounted on glass slides, air dried at $56^{\circ} \mathrm{C}$ overnight and subjected to immunostaining using an automated platform (Ventana Discovery XT, Tucson, AZ, USA). The following primary antibodies and dilutions were used: pERK1/2 1:300, pAkt(Ser473) 1:30, pEGFR 1:110, pmTOR 1:130, pHer3 1:70 (all from Cell Signaling Technology Inc., Danvers, MA, USA). After staining, sections were treated with ascending ethanol concentrations and xylene and were finally covered with Pertex (Medite $\mathrm{GmbH}$, Burgdorf, Germany). Sections were examined under a light microscope by a pathologist. Tumor cell staining was classified as absent, weak, moderate, or strong and a staining score was calculated based on the extent of staining according to the formula: score $=3 \times$ percentage of strongly stained tumor cells $+2 \mathrm{x}$ percentage of moderately stained tumor cells $+1 \mathrm{x}$ percentage of weakly stained tumor cells. All IHC staining protocols used within this study are "fit for purpose" and validated according to FDA guidelines, and staining procedures were also conducted according to standard operating procedures. Therefore, assays are controlled in terms of specificity and accuracy.

\section{Gene expression analysis}

For gene expression analysis, total RNA was extracted in duplicates from every frozen tissue block. Briefly, tissues were homogenized and RNA was isolated in two steps using phenol chloroform extraction and the RNeasy MinElute Cleanup Kit (Qiagen, Hilden, Germany) according to the manufacturer's instructions. RNA quality was evaluated based on $18 \mathrm{~S}$ and $28 \mathrm{~S}$ ribosomal RNA peaks using the Agilent 2100 bioanalyzer (Agilent Technologies, Berlin, Germany). Only RNA samples 
with an RNA integrity number $>7$ were used for gene expression analysis. RNA samples were analyzed in replicates using oligonucleotide microarrays (GeneChip Human Genome U133 Plus 2.0) based on the Affymetrix GeneChip $^{\mathrm{TM}}$ technology (Affymetrix Inc., Santa Clara, CA, USA).

\section{Statistical analysis}

Statistical analysis of protein content measured on the MSD platform and statistical analysis of staining scores derived from immunohistochemistry was performed with the Kruskal-Wallis test and Dunn's multiple comparisons test, using the software system GraphPad Prism Version 5.0 (GraphPad Software, San Diego, CA, USA). The significance level was 0.05 .

Statistical analysis of changes in gene expression was preceded by normalization using Affymetrix-PowerTools and $\log 2$ transformation. Symmetry of the data was verified by mean versus average analysis and boxplots. As a first approach towards statistical data analysis, hierarchical clustering was performed using Spearman correlation coefficient as distance measurement. The wardlinkage method was used to generate dendrograms. Since technical replicates were clustered together in almost all cases, these replicates were averaged and the average was used in further condition clustering, separating samples from normal tissue and those that originated from tumors. Thereafter, separate data across all four timepoints was clustered per individual patient, allowing patients to be grouped into distinct partitions.

Separating normal colon tissue from CRC tissue samples, only patients from the largest partition group were used to compare intensity levels between presurgery and $10^{\prime}$ postsurgery timepoints and presurgery and 45' postsurgery timepoints. T-tests were performed and p-values were calculated. Probe sets with $p>0.001$ were excluded as were probe sets with $<2$ log-fold changes in intensity. The overlap from both comparisons was identified as a list of genes that were particularly vulnerable to warm and cold ischemia. A list of all genes sorted according to the smallest coefficient of variation (CV) was used to identify genes that were apparently not vulnerable to warm and cold ischemia.

For detailed comparison between the three different cold ischemia time intervals, all samples taken at biopsy were excluded from the colorectal data pool. After exclusion, cluster dendrograms across the remaining three timepoints were generated, allowing patients to be grouped into distinct partitions. Patients from the largest partition group were used to compare intensity levels between presurgery and 10' postsurgery timepoint and presurgery and $45^{\prime}$ postsurgery timepoint. T-tests were performed and $\mathrm{p}$-values were calculated.

\section{ACKNOWLEDGMENTS}

Medical editing services were provided by Rhian Harper-Owen on behalf of Complete Medical Communications, funded by Indivumed.

\section{Competing financial interests}

This study was performed under the control of Indivumed; Kerstin A. David, Florian T. Unger, Philipp Uhlig and Hartmut Juhl are employees of Indivumed. The authors declare no other competing financial interests.

\section{Disclaimer}

This project was funded in part with Federal funds from the National Cancer Institute, National Institutes of Health, under contract HHSN2612008000001E. The content of this publication does not necessarily reflect the views or policies of the Department of Health and Human Services, nor does mention of trade names, commercial products, or organizations imply endorsement by the U.S. Government.

\section{REFERENCES}

1. Zatloukal K, Hainaut P. Human tissue biobanks as instruments for drug discovery and development: impact on personalized medicine. Biomark Med. 2010; 4: 895-903.

2. Vaught J, Lockhart NC. The evolution of biobanking best practices. Clin Chim Acta. 2012; 413: 1569-1575.

3. Juhl H. Preanalytical aspects: a neglected issue. Scand J Clin Lab Invest Suppl. 2010; 242: 63-65.

4. Huang J, Qi R, Quackenbush J, Dauway E, Lazaridis E, Yeatman T. Effects of ischemia on gene expression. J Surg Res. 2001; 99: 222-227.

5. Spruessel A, Steimann G, Jung M, Lee SA, Carr T, Fentz AK, Spangenberg J, Zornig C, Juhl HH, David KA. Tissue ischemia time affects gene and protein expression patterns within minutes following surgical tumor excision. BioTechniques. 2004; 36: 1030-1037.

6. Espina V, Edmiston KH, Heiby M, Pierobon M, Sciro M, Merritt B, Banks S, Deng J, VanMeter AJ, Geho DH, Pastore L, Sennesh J, Petricoin EF 3rd, Liotta LA. A portrait of tissue phosphoprotein stability in the clinical tissue procurement process. Mol Cell Proteomics. 2008; 7: 1998-2018.

7. Hatzis C, Sun H, Yao H, Hubbard RE, Meric-Bernstam F, Babiera GV, Wu Y, Pusztai L, Symmans WF. Effects of tissue handling on RNA integrity and microarray measurements from resected breast cancers. J Natl Cancer Inst. 2011; 103: 1871-1883.

8. Gündisch S, Hauck S, Sarioglu H, Schott C, Viertler C, 
Kap M, Schuster T, Reischauer B, Rosenberg R, Verhoef C, Mischinger HJ, Riegman P, Zatloukal K, Becker KF. Variability of protein and phosphoprotein levels in clinical tissue specimens during the preanalytical phase. J Proteome Res. 2012; 11: 5748-5762.

9. Rienzo M, Schiano C, Casamassimi A, Grimaldi V, Infante T, Napoli C. Identification of valid reference housekeeping genes for gene expression analysis in tumor neovascularization studies. Clin Transl Oncol. 2013; 15: 211-218.

10. Benndorf R, Bielka H. Cellular stress response: stress proteins--physiology and implications for cancer. Recent Results Cancer Res. 1997; 143: 129-144.

12. Landry J, Chrétien P, Laszlo A, Lambert H. Phosphorylation of HSP27 during development and decay of thermotolerance in Chinese hamster cells. J Cell Physiol. 1991; 147: 93-101.

13. Tai TC, Wong-Faull DC, Claycomb R, Wong DL. Hypoxic stress-induced changes in adrenergic function: role of HIF1 alpha. J Neurochem. 2009; 109: 513-524.

14. Jewell, UR, Kvietikova I, Scheid A, Bauer C, Wenger RH, Gassmann M. Induction of HIF-1 $\alpha$ in response to hypoxia is instantaneous. FASEB J. 2001; 15: 1312-1314.

15. Lau LF. CCN1/CYR61: the very model of a modern matricellular protein. Cell Mol Life Sci. 2011; 68: 3149 3163.

16. Bansal G, Druey KM, Xie Z. R4 RGS proteins: regulation of G-protein signaling and beyond. Pharmacol Ther. 2007; 116: 473-495.

17. Lawan A, Shi H, Gatzke F, Bennett AM. Diversity and specificity of the mitogen-activated protein kinase phosphatase-1 functions. Cell Mol Life Sci. 2013; 70, 223 237.

18. Maltseva DV, Khaustova NA, Fedotov NN, Matveeva EO, Lebedev AE, Shkurnikov MU, Galatenko VV, Schumacher U, Tonevitsky AG. High-throughput identification of reference genes for research and clinical RT-qPCR analysis of breast cancer samples. J Clin Bioinforma. 2013; 22: 13. 